\title{
Effective field theory of the classical two-dimensional plasma
}

\author{
M A Valle Basagoiti \\ Departamento de Física Teórica, Universidad del País Vasco, Apartado 644, E-48080 \\ Bilbao, Spain \\ E-mail: wtpvabam@lg.ehu.es
}

\begin{abstract}
Using techniques of effective field theory, we consider the thermodynamical properties of a dilute two-dimensional plasma interacting via a $1 / r$ potential. The first one-loop correction to the partition function is already logarithmically divergent in the effective theory. The finite part of the one-loop induced couplings is then explicitly evaluated by matching the density-density correlator in the effective theory and in the full quantum theory. This task is performed from the formulation of the Coulomb problem in momentum space by projecting the two-dimensional momentum space onto the surface of a three-dimensional sphere. We also report some higher order results that, in the case of the one-component plasma immersed in a uniform neutralizing background, are resummed to obtain the complete leading-log partition function.
\end{abstract}

Submitted to: J. Phys. A: Math. Gen.

PACS numbers: 11.10.Kk, 52.27.-h, 3.65.-w 


\section{Introduction}

An application of effective field theory methods, which have become commonplace in high-energy physics and nuclear physics, has been achieved very recently by Brown and Yaffe [1] to analyze some general equilibrium properties of non-relativistic classical plasmas. In an exhaustive work, these authors have systematically derived a couple of higher-order results concerning with the thermodynamics and have computed some correlation functions of the three-dimensional classical plasma. Although of some of those results were known, the computation is streamlined by the organization inherent in the technique of effective interactions.

The two-dimensional electron gas with $1 / r$ interactions is both a fundamental model in many-body physics and a system of current experimental interest. Electrons trapped on the liquid-helium surface or electrons confined in the vicinity of a junction between a semiconductor and insulators or between layers of different semiconductors are examples of two-dimensional electron systems [2]. At finite temperature, the parameter $n \lambda^{2}$ measures the dilution of the system and the dimensionless coupling parameter defined by $g=2 \pi n \beta^{2} e^{4}$ characterizes the strength of Coulomb interactions, where $\lambda$ denotes the thermal wavelength and $e$ is the effective electronic charge in unrationalized units. For electrons on a helium film the electron density can be varied in the range from $10^{5}$ $\mathrm{cm}^{-2}$ to $10^{9} \mathrm{~cm}^{-2}$. For these low densities, it appears that the electrons form classical two-dimensional systems even at temperatures of a few millidegrees Kelvin. For $T \sim 1$ $\mathrm{K}$ and $10^{5}<n<10^{6} \mathrm{~cm}^{-2}$, the range of the plasma parameter is $1.7<g<17$, not very far from the domain of weak coupling.

In this paper, we largely pursue the methods advocated by Brown and Yaffe, and consider a classical two-dimensional multi-component plasma interacting via the Coulomb potential. From the appropriate scalar field theory, we compute the partition function and the correlation functions to first nontrivial order requiring renormalization. The analytical results that we have derived are valid in the low density and weak coupling domain where $n \lambda^{2} \ll 1$ and $g \ll 1$. The essential ingredients in the calculation are the matching coefficients or induced couplings, whose finite parts are determined from a comparison of the short-distance behaviours in the effective theory and in the full quantum theory. The main result of this paper is precisely the computation of the matching coefficients, exploiting some properties in the momentum space of the Coulomb Green's function for the quantum problem.

There are old analytical and numerical results on the two-dimensional onecomponent plasma in the classical regime [3, 4, 5, 6. Chalupa [4] reports an equation of state given by

$$
\frac{\beta p}{n}-1=\frac{g}{4} \ln g+\frac{g}{4}\left(2 \gamma_{\mathrm{E}}-1+\ln 2\right)+O\left(g^{2} \ln ^{2} g, g^{2} \ln g, g^{2}\right),
$$

and Totsuji [5] gives the ratio of the correlation energy density to the kinetic energy as

$$
\frac{\beta E_{\mathrm{c}}}{n}=\frac{\beta u}{n}-1=\frac{g}{2} \ln g+\frac{g}{2}\left(2 \gamma_{\mathrm{E}}-1+\ln 2\right)+\ldots
$$


These expressions agree precisely with our results (38) and (40) for the pressure and the internal energy when the ratio of the Coulomb energy of a pair of charged particles separated by a thermal length to the thermal energy is very large, $\eta=\beta e^{2} / \lambda \gg 1$. However, as we will show below, our one-loop results do not imply restriction to this ratio. They are valid to order $g$ for arbitrary $\eta$. The terms of order $g^{2}$ retained by Chalupa and Totsuji are not reliable because they must be computed by matching with unknown results of a three-body calculation in the quantum theory. However, we have been able to compute all the $g^{n}(\ln g)^{n}$ leading logarithmic terms of the partition function in (57).

The plan of this paper is arranged as follows. In section 2 we briefly review the structure of the effective theory, the partition function to one-loop order and write down the form of the induced couplings. In Section 3, with the matching procedure completed, we compute at one-loop level the equation of state, the internal energy and the number density correlators. The section 4 is devoted to report some higher order results concerning the leading logarithmic contribution to the partition function. A short conclusion is presented in section 5. Details of the derivation of the basic formulae for the matching procedure are given in the appendix.

\section{One-loop divergences in the effective theory}

We consider a plasma of different species of charged particles interacting through the Coulomb interaction. The charge, mass, .. of the species $a$ are denoted by $e_{a}, m_{a}, \ldots$ These are the conventions of 1 which we shall closely follow. In the classical limit, the free-particle distribution function reduces to a gaussian function $f_{a}^{0}(\boldsymbol{p})=e^{\beta \mu_{a}} e^{-\beta p^{2} / 2 m_{a}}$, and the free-particle density in two dimensions is $n_{a}^{0}=g_{a} \lambda_{a}^{-2} e^{\beta \mu_{a}}$, where

$$
\lambda_{a}=\left(\frac{2 \pi \beta \hbar^{2}}{m_{a}}\right)^{1 / 2}
$$

denotes the thermal wavelength of the species $a$ and $g_{a}, \mu_{a}$ are the corresponding degeneracy and chemical potential. We shall assume that the system is electrically neutral.

The Fourier transform of the $1 / r$ potential in two dimensions is

$$
\int d^{2} \boldsymbol{r} \frac{e^{-i \boldsymbol{k} \cdot \boldsymbol{r}}}{r}=\frac{2 \pi}{k}
$$

which gives rise to the interaction potential in $\nu$ spatial dimensions

$$
V_{\nu}\left(\boldsymbol{r}-\boldsymbol{r}^{\prime}\right)=\int \frac{d^{\nu} \boldsymbol{k}}{(2 \pi)^{\nu}} \frac{e^{i \boldsymbol{k} \cdot\left(\boldsymbol{r}-\boldsymbol{r}^{\prime}\right)} 2 \pi}{k}=\Gamma\left(\frac{\nu-1}{2}\right) \pi^{(1-\nu) / 2}\left|\boldsymbol{r}-\boldsymbol{r}^{\prime}\right|^{1-\nu}
$$

As usual, in the absence of any scale, the analytically continued coincidence limit $V_{\nu}(\mathbf{0})$ will be taken as zero. Using the fact that $\sqrt{-\nabla^{2}} /(2 \pi)$ is the inverse operator of $1 / r$ in two dimensions, the grand canonical partition function in the classical limit can be turned via a Hubbard-Stratonovich transformation into the functional integral

$$
\mathcal{Z}(\mu)=\operatorname{Det}^{1 / 2}\left[\frac{\beta \sqrt{-\nabla^{2}}}{2 \pi}\right] \int \mathcal{D} \phi(\boldsymbol{r}) \exp \left(-S_{\mathrm{cl}}[\phi ; \mu]\right),
$$


with the action functional of the electrostatic potential $\phi$ defined by

$$
S_{\mathrm{cl}}[\phi ; \mu]=\int d^{2} \boldsymbol{r}\left\{\frac{\beta}{2} \phi(\boldsymbol{r}) \frac{\sqrt{-\nabla^{2}}}{2 \pi} \phi(\boldsymbol{r})-\sum_{a} n_{a}^{0}(\boldsymbol{r}) e^{i \beta e_{a} \phi(\boldsymbol{r})}\right\} .
$$

where $n_{a}^{0}(\boldsymbol{r})=g_{a} \lambda_{a}^{-2} e^{\beta \mu(\boldsymbol{r})}$. The corresponding field equation is the analog of the DebyeHückel result in two dimensions. Here, it proves convenient to assume that the chemical potentials entering into $n_{a}^{0}$ can depend upon $\boldsymbol{r}$. Thus, the functional derivation with respect to $\mu_{a}(\boldsymbol{r})$ will produce the correlation function between densities

$$
K_{a b}\left(\boldsymbol{r}-\boldsymbol{r}^{\prime}\right)=\frac{\delta^{2} \ln \mathcal{Z}(\mu)}{\delta \beta \mu_{a}(\boldsymbol{r}) \delta \beta \mu_{b}\left(\boldsymbol{r}^{\prime}\right)} .
$$

Since the total charge neutrality in terms of the free-particle densities is

$$
\sum_{a} e_{a} n_{a}^{0}=0
$$

the $\phi=0$ configuration is a solution of the classical field equation and, consequently, the functional integral can be perturbatively computed from a saddle point expansion around this trivial solution. The quadratic part of the action,

$$
S_{0}[\phi ; \mu]=\int d^{2} \boldsymbol{r}\left\{-\sum_{a=1}^{A} n_{a}^{0}+\frac{\beta}{2} \phi(\boldsymbol{r})\left[\frac{\sqrt{-\nabla^{2}}}{2 \pi}+\frac{\kappa_{0}}{2 \pi}\right] \phi(\boldsymbol{r})\right\},
$$

includes the inverse Debye length, $\kappa_{0} \equiv 2 \pi \beta \sum_{a} e_{a}^{2} n_{a}^{0}$, and the remaining part giving rise to the perturbative expansion is

$$
\Delta S[\phi ; \mu]=-\int d^{2} \boldsymbol{r} \sum_{a=1}^{A} n_{a}^{0}\left\{e^{i \beta e_{a} \phi(\boldsymbol{r})}-1+\frac{1}{2} \beta^{2} e_{a}^{2} \phi(\boldsymbol{r})^{2}\right\} .
$$

This effective field theory can be systematically obtained from the quantum statistical mechanics of the plasma by integration of the quantum fields with momentum scales of order $\lambda^{-1}$ or larger [1]. In addition to the terms already present in (7), there are sub-leading contributions in a derivative expansion in powers of $(\lambda \partial \phi)$. The first of these comes from the static non-interacting two-point charge density-charge density correlation at non-vanishing $\boldsymbol{k}$ and zero frequency, evaluated within the MaxwellBoltzmann statistics. This reads

$$
\Pi(\boldsymbol{k}, \omega=0)=\frac{\kappa_{0}}{2 \pi}\left(1-\frac{\lambda^{2} k^{2}}{24 \pi}+\ldots\right),
$$

where the piece independent upon $\boldsymbol{k}$ produces the Debye screening included in (17) and the next term yields an action contribution linear in the particle densities,

$$
-\int d^{2} \boldsymbol{r} \frac{\beta \kappa_{0} \lambda^{2}}{96 \pi^{2}} \nabla \phi(\boldsymbol{r}) \cdot \nabla \phi(\boldsymbol{r})
$$

This action must be made consistent with the invariance of the original theory under the simultaneous constant shift $\phi \rightarrow \phi-i c$ and $\mu_{a} \rightarrow \mu_{a}-e_{a} c$. Consequently, the corresponding derivative interaction, linear in the particle densities must have the form

$$
S_{\text {ind }}^{(1)}[\phi ; \mu]=\sum_{a} \int d^{2} \boldsymbol{r} \frac{\beta^{2} \lambda_{a}^{2}}{48 \pi}\left[\nabla\left(\mu_{a}(\boldsymbol{r})+i e_{a} \phi(\boldsymbol{r})\right)\right]^{2} n_{a}^{0}(\boldsymbol{r}) e^{i \beta e_{a} \phi(\boldsymbol{r})},
$$


where we have used the form of the Debye wave number.

In the following, we safely use the effective theory of equation (77) to describe distance scales of order $\lambda$ and larger. Although this classical regime is independent of the quantum statistics, the logarithmic ultraviolet divergences in the effective theory are related to quantum properties of the full theory. These divergences of the form $\ln \left(\kappa_{0} / \mu\right)$, where $\mu$ is a momentum scale introduced by dimensional regularization, must match onto counterterms proportional to $\ln (\mu \lambda)$ arising from the action with the induced couplings required to cancel the $\mu$ dependence. The precise value of the finite part of the induced couplings is determined by comparison with the result of the computation of some quantity in the full quantum theory. The main result of this work is justly the explicit evaluation of the one-loop induced couplings.

The dimensionless loop expansion parameter is the ratio of the Coulomb energy for two particles separated by a Debye length to their average kinetic energy in the plasma. This plasma parameter is $g=\beta e^{2} \kappa_{0}$. Apart from the Debye length, there is another relevant length scale, not directly entering into the effective theory, but playing an important role in the matching procedure. This is the Bohr radius $\left(e^{2} m_{a b}\right)^{-1}$ or, equivalently, the binding energy $e^{4} m_{a b} / 2$ of two particles in the plasma with reduced mass $m_{a b}$ and equal and opposite charge.

The Green's function $G_{\nu}\left(\boldsymbol{r}-\boldsymbol{r}^{\prime}\right)$ in $\nu$ dimensions, including the effects of static screening, is given in terms of Struve functions $\mathbf{H}$ and Bessel functions of second kind $Y$ by

$$
G_{\nu}\left(\boldsymbol{r}-\boldsymbol{r}^{\prime}\right)=\int \frac{d^{\nu} \boldsymbol{k}}{(2 \pi)^{\nu}} \frac{e^{i \boldsymbol{k} \cdot\left(\boldsymbol{r}-\boldsymbol{r}^{\prime}\right)} 2 \pi}{k+\kappa_{0}}=V_{\nu}\left(\boldsymbol{r}-\boldsymbol{r}^{\prime}\right)+\Delta V_{\nu}\left(\left|\boldsymbol{r}-\boldsymbol{r}^{\prime}\right|\right)
$$

with

$$
\Delta V_{\nu}(r)=\frac{2^{-\nu / 2} \pi^{2-\nu / 2} \kappa_{0}^{\nu / 2} r^{1-\nu / 2}}{\cos (\pi \nu / 2)}\left[\mathbf{H}_{1-\nu / 2}\left(\kappa_{0} r\right)-Y_{1-\nu / 2}\left(\kappa_{0} r\right)\right],
$$

and the coincidence limit is

$$
G_{\nu}(\mathbf{0})=\frac{2^{2-\nu} \pi^{2-\nu / 2} \kappa_{0}^{\nu-1}}{\Gamma(\nu / 2) \sin \pi \nu}
$$

With these results in hand, the one-loop contribution to $\ln \mathcal{Z} / \mathcal{A}$ for the effective theory is given by

$$
\begin{aligned}
\frac{\ln \mathcal{Z}}{\mathcal{A}} & =\sum_{a} n_{a}^{0}-\frac{1}{2 \mathcal{A}} \ln \operatorname{Det}\left[1+\frac{1}{\sqrt{-\nabla^{2}}} \kappa_{0}\right] \\
& =\sum_{a} n_{a}^{0}-\frac{\kappa_{0}}{4 \pi \nu} G_{\nu}(\mathbf{0}),
\end{aligned}
$$

where the determinant has been evaluated by integration of the formula

$$
\delta \ln \operatorname{Det}\left[1+\frac{1}{\sqrt{-\nabla^{2}}} \kappa_{0}\right]=\int d^{\nu} \boldsymbol{r} G_{\nu}(\mathbf{0}) \frac{\delta \kappa_{0}}{2 \pi} .
$$

This contribution is singular when $\nu \rightarrow 2$ and, already to this order, renormalization is required. To determine the form of counterterms, we note that the pole term is 


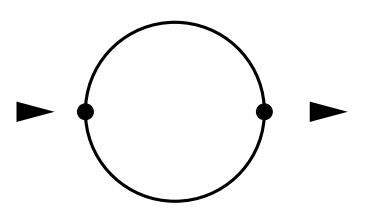

a

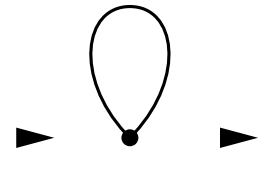

$\mathrm{b}$

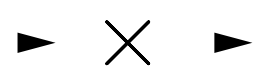

C

Figure 1. One-loop diagrams contributing to the irreducible part $\widetilde{C}_{a b}(\boldsymbol{k})$ of the density-density correlator. The diagram c represents the contribution of induced couplings.

proportional to $\kappa_{0}^{2}$. Thus, at this order, the induced couplings must be quadratic in the particle densities. The counterterm action, to be handled at tree level to cancel the one-loop divergences, must have the form [1]

$$
S_{\text {ind }}^{(2)}[\phi ; \mu]=\sum_{a, b} \int d^{\nu} \mathbf{r} \beta^{2} g_{a b}^{0} n_{a}^{0} n_{b}^{0} e^{i \beta e_{a} \phi(\mathbf{r})} e^{i \beta e_{b} \phi(\mathbf{r})},
$$

where the pole term of $g_{a b}^{0}$

$$
g_{a b}^{0}=\mu^{\nu-2}\left[\frac{\pi e_{a}^{2} e_{b}^{2}}{2} \frac{1}{2-\nu}+g_{a b}(\mu)\right],
$$

cancels the $\nu \rightarrow 2$ pole of $\ln \mathcal{Z}$ coming from $G_{2}(\mathbf{0})$. The momentum scale $\mu$ is required in order for the finite part of the coupling $g_{a b}(\mu)$ preserve its dimensions at $\nu=2$ when $\nu$ is arbitrary. To this order, the contribution of the induced couplings only involves the $\phi=0$ part of $S_{\text {ind }}$ but at higher orders, when it is neccesary to handle the induced interaction beyond the tree level, the full exponential dependence upon $\phi$ will be crucial.

The renormalization group equation for $g_{a b}(\mu)$,

$$
\mu \frac{d}{d \mu} g_{a b}(\mu)=\frac{\pi e_{a}^{2} e_{b}^{2}}{2}
$$

guarantees that the bare induced couplings $g_{a b}^{0}$ do not depend on $\mu$. By integration, one obtain

$$
g_{a b}(\mu)=\frac{\pi e_{a}^{2} e_{b}^{2}}{4} \ln \left(\frac{\mu^{2}}{\mu_{a b}^{2}}\right)
$$

where the integration constant $\mu_{a b}$ cannot be determined within the effective theory. The simplest way to determine the short-distance parameter $\mu_{a b}$ is to match the quadratic part in the particle densities of the one-loop density-density correlator $\widetilde{K}_{a b}(\boldsymbol{k})$ in the effective theory and in the full quantum theory [1. In this comparison of the shortdistance behaviours, the Debye screening plays no role [9] provided that the correlators are evaluated at non-zero wave number $\boldsymbol{k}$.

As discussed by Brown and Yaffe [1], the Fourier transform of the complete densitydensity correlator has the general form

$$
\widetilde{K}_{a b}(\boldsymbol{k})=\widetilde{C}_{a b}(\boldsymbol{k})-\frac{\left(\beta \sum_{c} e_{c} \widetilde{C}_{c a}(\boldsymbol{k})\right) 2 \pi\left(\beta \sum_{c} e_{c} \widetilde{C}_{c b}(\boldsymbol{k})\right)}{\beta\left(|\boldsymbol{k}|+2 \pi \beta \sum_{m n} e_{m} e_{n} \widetilde{C}_{m n}(\boldsymbol{k})\right)}
$$


Here, $\widetilde{C}_{a b}(\boldsymbol{k})$ denotes the Fourier transform of

$$
C_{a b}\left(\boldsymbol{r}-\boldsymbol{r}^{\prime}\right)=-\left.\frac{\delta^{2} \Gamma[\bar{\phi} ; \mu]}{\delta \beta \mu_{a}(\boldsymbol{r}) \delta \beta \mu_{b}\left(\boldsymbol{r}^{\prime}\right)}\right|_{\bar{\phi}=0},
$$

where $\Gamma[\bar{\phi} ; \mu]$ is the effective action of the theory. At tree level, $\Gamma[\bar{\phi} ; \mu]=S_{\mathrm{cl}}[\bar{\phi} ; \mu]$ and, consequently, $\widetilde{C}_{a b}^{\text {tree }}(\boldsymbol{k})=\delta_{a b} n_{a}^{0}$ (no sum over $a$ ). Noting that $S_{\text {ind }}^{(1)}[\phi ; \mu]+S_{\text {ind }}^{(2)}[\phi ; \mu]$ in equations (14) and (20) must be handled at tree level, the contribution to $\widetilde{C}_{a b}(\boldsymbol{k})$ up to one-loop order is given by

$$
\begin{aligned}
\widetilde{C}_{a b}^{(1)}(\boldsymbol{k})= & \delta_{a b} n_{b}^{0}+\beta^{2} n_{a}^{0} n_{b}^{0}\left[\frac{e_{a}^{2} e_{b}^{2}}{2} D_{\nu}^{(2)}(\boldsymbol{k})-2 g_{a b}^{0}\right]-\frac{1}{2} \beta \delta_{a b} e_{b}^{2} n_{b}^{0} G_{\nu}(\mathbf{0}) \\
& -2 \beta^{2} \delta_{a b} \sum_{c} g_{b c}^{0} n_{b}^{0} n_{c}^{0}-\frac{\lambda_{a}^{2} k^{2}}{24 \pi} \delta_{a b} n_{b}^{0}
\end{aligned}
$$

where

$$
D_{\nu}^{(2)}(\boldsymbol{k}) \equiv \int d^{\nu} \boldsymbol{r} e^{-i \boldsymbol{k} \cdot \boldsymbol{r}} G_{\nu}(\boldsymbol{r})^{2}
$$

The function $D_{\nu}^{(2)}(\boldsymbol{k})$ comes from the one-loop diagram a in figure 1 and $G_{\nu}(\mathbf{0})$ is the tadpole. Since the last term of equation (26) yields a contribution to the $n_{a}^{0} n_{b}^{0}$ part of $\widetilde{K}_{a b}(\boldsymbol{k})$ which vanishes linearly when $k \rightarrow 0$, this term can be ignored in the matching procedure at small momentum $\boldsymbol{k}$. Therefore, ignoring Debye screening,

$D_{\nu}^{(2)}(\boldsymbol{k}) \rightarrow C_{\nu}^{(2)}(\boldsymbol{k}) \equiv \int d^{\nu} \boldsymbol{r} e^{-i \boldsymbol{k} \cdot \boldsymbol{r}} V_{\nu}(\boldsymbol{r})^{2}=\frac{2^{2-\nu} \pi^{1-\frac{\nu}{2}} \Gamma\left(1-\frac{\nu}{2}\right) \Gamma\left(\frac{1-\nu}{2}\right)^{2}}{\Gamma(\nu-1)} k^{\nu-2}$

This replacement leads to the piece of the correlator to be compared to the corresponding result in the full quantum theory

$$
\begin{aligned}
\widetilde{K}_{a b}^{\text {quad }}(\boldsymbol{k})= & \beta^{2} n_{a}^{0} n_{b}^{0}\left[\frac{e_{a}^{2} e_{b}^{2}}{2} C_{\nu}^{(2)}(\boldsymbol{k})-2 g_{a b}^{0}\right]-\frac{2 \pi \beta n_{a}^{0} n_{b}^{0} e_{a} e_{b}}{k} \\
& =-\beta^{2} n_{a}^{0} n_{b}^{0}\left\{\frac{\pi e_{a}^{2} e_{b}^{2}}{2}\left[\gamma_{\mathrm{E}}+\ln \left(\frac{k^{2}}{64 \pi \mu^{2}}\right)\right]+2 g_{a b}(\mu)\right\}-\frac{2 \pi \beta n_{a}^{0} n_{b}^{0} e_{a} e_{b}}{k} .
\end{aligned}
$$

\section{Matching and one-loop complete results}

In order to perform the matching, we consider the $\boldsymbol{k} \rightarrow 0$ behaviour of the secondorder contribution in the fugacity expansion to the quantum mechanical density-density correlator $\mathcal{K}_{a b}(\boldsymbol{k})$. The details of the computation are given in the appendix. This contribution to the full correlator is

$$
\mathcal{K}_{a b}^{(2)}(\boldsymbol{k})=n_{a}^{0} n_{b}^{0} \lambda_{a b}^{2}\left[F_{+}(\boldsymbol{k}) \pm\left(\delta_{a b} / g_{a}\right) F_{-}(\boldsymbol{k})\right],
$$

where the \pm sign accounts for Bose $(+)$ or Fermi $(-)$ statistics, $g_{a}$ denotes the spin degeneracy of the species $a$, and $\lambda_{a b}$ is the thermal wavelength for the reduced mass $m_{a b}=m_{a} m_{b} /\left(m_{a}+m_{b}\right)$. The required terms are (Fourier transformed) matrix elements of the heat kernel constructed in terms of the Hamiltonian $H$ for relative motion, and read

$$
F_{ \pm}(\boldsymbol{k})=\int d^{2} \boldsymbol{r} e^{-i \boldsymbol{k} \cdot \boldsymbol{r}}\left\langle\boldsymbol{r}\left|e^{-\beta H_{a b}}\right| \pm \boldsymbol{r}\right\rangle
$$


with

$$
H_{a b}=\frac{\boldsymbol{p}^{2}}{2 m_{a b}}+\frac{e_{a} e_{b}}{r} .
$$

With the results (A.29) and (A.48) in hand, the comparison of (30) with (29) enables to determine the integration constant $\mu_{a b}$ in the running coupling

$$
g_{a b}(\mu)=\frac{\pi e_{a}^{2} e_{b}^{2}}{4}\left[\ln \left(\mu^{2} \lambda_{a b}^{2}\right)+f\left(\eta_{a b}\right) \pm\left(\delta_{a b} / g_{a}\right) \tilde{f}\left(\eta_{a a}\right)\right],
$$

in terms of the quantum parameter $\eta_{a b}=\beta e_{a} e_{b} / \lambda_{a b}$. Thereby, the complete contribution to $\ln \mathcal{Z}$ up to one-loop order in terms of the fugacities is

$$
\frac{\ln \mathcal{Z}(\mu)}{\mathcal{A}}=\sum_{a} n_{a}^{0}-\frac{\pi}{4} \sum_{a, b} n_{a}^{0} n_{b}^{0}\left(\beta e_{a} e_{b}\right)^{2}\left[\ln \left(\frac{\lambda_{a b}^{2} \kappa_{0}^{2}}{4 \pi}\right)-1+\gamma_{\mathrm{E}}+\Gamma_{a b}\right]
$$

where

$$
\Gamma_{a b}=f\left(\eta_{a b}\right) \pm\left(\delta_{a b} / g_{a}\right) \tilde{f}\left(\eta_{a a}\right) .
$$

By inversion of the formula

$$
\bar{n}_{a}=\frac{1}{\beta} \frac{\partial}{\partial \mu_{a}} \frac{\ln \mathcal{Z}(\mu)}{\mathcal{A}},
$$

the elimination of the chemical potentials in favour of the mean number densities $\bar{n}_{a}$ can be made perturbatively, and at one-loop order we obtain

$$
n_{a}^{0}=\bar{n}_{a}\left(1+\frac{\pi}{2} \sum_{b} \bar{n}_{b}\left(\beta e_{a} e_{b}\right)^{2}\left[\ln \left(\frac{\lambda_{a b}^{2} \bar{\kappa}_{0}^{2}}{4 \pi}\right)+\gamma_{\mathrm{E}}+\Gamma_{a b}\right]\right),
$$

with $\bar{\kappa}_{0}=2 \pi \beta \sum_{a} e_{a}^{2} \bar{n}_{a}$. The corresponding replacement in the expression for the pressure $p=\ln \mathcal{Z} / \beta$ gives the equation of state

$$
\beta p=\sum_{a} \bar{n}_{a}+\frac{\pi}{4} \sum_{a, b} \bar{n}_{a} \bar{n}_{b}\left(\beta e_{a} e_{b}\right)^{2}\left[\ln \left(\frac{\lambda_{a b}^{2} \bar{\kappa}_{0}^{2}}{4 \pi}\right)+1+\gamma_{\mathrm{E}}+\Gamma_{a b}\right] .
$$

The internal energy $u$ density is given by

$$
u=-\frac{\partial}{\partial \beta} \frac{\ln \mathcal{Z}(\mu)}{\mathcal{A}}+\sum_{a} \mu_{a} \bar{n}_{a}
$$

which yields

$\beta u=\sum_{a} \bar{n}_{a}+\frac{\pi}{2} \sum_{a, b} \bar{n}_{a} \bar{n}_{b}\left(\beta e_{a} e_{b}\right)^{2}\left[\ln \left(\frac{\lambda_{a b}^{2} \bar{\kappa}_{0}^{2}}{4 \pi}\right)+\frac{1}{2}+\gamma_{\mathrm{E}}+\Gamma_{a b}+\frac{1}{4} \eta_{a b} \Gamma_{a b}^{\prime}\right]$,

where

$$
\Gamma_{a b}^{\prime}=f^{\prime}\left(\eta_{a b}\right) \pm\left(\delta_{a b} / g_{a}\right) \tilde{f}^{\prime}\left(\eta_{a a}\right) .
$$

In the case of a plasma consisting of a single species immersed in a uniform neutralizing background, the terms of order $\epsilon^{2}$ and $\epsilon^{2} \ln \epsilon$ which appear in the equation of state reported by Chalupa 44 agree precisely with our result (38) when the strong repulsion result (A.39) is inserted $\ddagger$ into (35), neglecting the term of order $\eta_{a a}^{-2}$ and the exchange term (A.50). The ratio of the correlation energy density to the kinetic energy density $\beta E_{\mathrm{c}} / n$ reported by Totsuji [5] also agrees with the term of order $e^{4}$ in (40) when $\eta_{a a} \gg 1$.

$\ddagger$ The parameter $\epsilon^{2}$ in $\left[4\right.$ is $4 \pi \bar{n} e^{4} \beta^{2}=2 \bar{\kappa}_{0} \lambda_{a a} \eta_{a a}$, and the parameter $\epsilon$ in [5] is $2 \pi \bar{n} e^{4} \beta^{2}$. 
Number density correlators

The insertion of our findings into (26) yields the irreducible part of the number density correlator function to one-loop order, in terms of bare densities

$$
\widetilde{C}_{a b}(\boldsymbol{k})=\frac{1}{2}\left(\beta e_{a}^{2} n_{a}^{0}\right) \widetilde{F}_{a b}^{0}(\boldsymbol{k})\left(\beta e_{b}^{2} n_{b}^{0}\right)+\delta_{a b} n_{a}^{0} \widetilde{F}_{a}^{0}(\boldsymbol{k})
$$

Using (17) and (21), the contact term reads

$$
\widetilde{F}_{a}^{0}(\boldsymbol{k})=1-\frac{\pi}{2} \sum_{b} n_{b}^{0}\left(\beta e_{a} e_{b}\right)^{2}\left[\ln \left(\frac{\lambda_{a b}^{2} \kappa_{0}^{2}}{4 \pi}\right)+\gamma_{\mathrm{E}}+\Gamma_{a b}\right]-\frac{\lambda_{a}^{2} k^{2}}{24 \pi},
$$

and the counterpart $\delta_{a b} \bar{n}_{a} \widetilde{F}_{a}(\boldsymbol{k})$ in terms of the mean number densities is readily written with the aid of (37),

$$
\widetilde{F}_{a}(\boldsymbol{k})=1-\frac{\lambda_{a}^{2} k^{2}}{24 \pi}\left(1+\frac{\pi}{2} \sum_{b} \bar{n}_{b}\left(\beta e_{a} e_{b}\right)^{2}\left[\ln \left(\frac{\lambda_{a b}^{2} \bar{\kappa}_{0}^{2}}{4 \pi}\right)+\gamma_{\mathrm{E}}+\Gamma_{a b}\right]\right) .
$$

Since $G_{2}(\boldsymbol{r})^{2}-V_{2}(\boldsymbol{r})^{2}$ behaves as $\ln \left(\kappa_{0} r\right) / r$ as $r \rightarrow 0$, the non-vanishing part of $D_{\nu}^{(2)}(\boldsymbol{k})$ when $\nu \rightarrow 2$ may be written as

$$
D_{\nu}^{(2)}(\boldsymbol{k})=C_{\nu}^{(2)}(\boldsymbol{k})+\Delta C_{2}^{(2)}(\boldsymbol{k}),
$$

where $\Delta C_{2}^{(2)}(\boldsymbol{k})$ is the Fourier transform of

$$
G_{2}(\boldsymbol{r})^{2}-V_{2}(\boldsymbol{r})^{2}=\Delta V_{2}(r)\left(\Delta V_{2}(r)-\frac{2}{r}\right),
$$

and the asymptotic behavior of $\Delta V_{2}(r)$ reads

$$
\Delta V_{2}(r)=\frac{\pi \kappa_{0}}{2}\left(Y_{0}\left(\kappa_{0} r\right)-\mathbf{H}_{0}\left(\kappa_{0} r\right)\right) \sim-\frac{1}{r}+\frac{1}{\kappa_{0}^{2} r^{3}}+\ldots
$$

With this definition, we obtain

$$
\widetilde{F}_{a b}^{0}(\boldsymbol{k})=\Delta C_{2}^{(2)}(\boldsymbol{k})-\pi\left[\ln \left(\frac{\lambda_{a b}^{2} k^{2}}{64 \pi}\right)+\gamma_{\mathrm{E}}+\Gamma_{a b}\right] .
$$

We must emphasize that the dependence upon $\ln \left(k^{2}\right)$ is deceptive. In fact, an explicit calculation when $\boldsymbol{k} \rightarrow 0$ shows that

$$
\Delta C_{2}^{(2)}(\boldsymbol{k})=-2 \pi\left[1-\ln \left(\frac{k}{4 \kappa_{0}}\right)\right]-\frac{\pi k^{2}}{\kappa_{0}^{2}} \ln \left(\frac{k}{\kappa_{0}}\right)+O\left(k^{2}\right),
$$

and, consequently

$$
\widetilde{F}_{a b}^{0}(\mathbf{0})=-\pi\left[\ln \left(\frac{\lambda_{a b}^{2} \kappa_{0}^{2}}{4 \pi}\right)+2+\gamma_{\mathrm{E}}+\Gamma_{a b}\right],
$$

in perfect agreement with the general result $\widetilde{C}_{a b}(\mathbf{0})=-\partial \bar{n}_{a} / \partial \beta \mu_{b}$. Note that, to oneloop order, the $n_{a}^{0} n_{b}^{0}$ piece of $\widetilde{C}_{a b}$ can be written in terms of the mean densities by replacing $n^{0} \rightarrow \bar{n}$. 


\section{Renormalization at higher order}

The complete determination of two-loop and higher order contributions to $\ln \mathcal{Z}$ requires at least to perform a difficult three-body calculation in the quantum theory. However, it is straighforward to derive renormalization group equations for the leading logarithmic pieces of the renormalized multiloop induced couplings $g_{i_{1} \ldots i_{n}}^{0}$ that enter into the counterm action through

$$
S_{\text {ind }}[\phi ; \mu]=\sum_{n=2} \sum_{i_{1} \ldots i_{n}} \int d^{\nu} \boldsymbol{r} g_{i_{1} \ldots i_{n}}^{0} \beta^{2(n-1)} n_{i_{1}}^{0} e^{i \beta e_{i_{1}} \phi} \ldots n_{i_{n}}^{0} e^{i \beta e_{i_{n}} \phi} .
$$

The coupling $g_{i_{1} \ldots i_{n}}^{0}$ is proportional to $e^{4(n-1)}$ since at $\nu=2$, all factors of $n_{i}^{0}$ excepting one of them must be accompanied by a factor of $\beta^{2} e^{4}$, in order to reproduce the right power counting. Thus, the induced coupling $g_{i_{1} \ldots i_{n}}^{0}$ first contributes to $\ln \mathcal{Z} / \mathcal{A}$ at order $n-1$ loop. This contribution is

$$
I_{g_{i_{1} \ldots i_{n}}}^{(n-1)}=-\beta^{2(n-1)} \sum_{i_{1} \ldots i_{n}} g_{i_{1} \ldots i_{n}}^{0} n_{i_{1}}^{0} \ldots n_{i_{n}}^{0} .
$$

Another contribution at order $(n-1)$-loop which arises from the one-loop graph with one insertion of the induced interaction $g_{i_{1} \ldots i_{n-1}}^{0}$ is

$$
\begin{aligned}
I_{g_{i_{1} \ldots i_{n-1}}}^{(n-1)}= & \beta^{2(n-1)-1} \sum_{i_{1} \ldots i_{n-1}} g_{i_{1} \ldots i_{n-1}}^{0} n_{i_{1}}^{0} \ldots n_{i_{n-1}}^{0}\left(e_{i_{1}}+\ldots+e_{i_{n-1}}\right)^{2} \frac{1}{2} G_{\nu}(\mathbf{0}) \\
& =\beta^{2(n-1)} \pi \kappa_{0}^{\nu-2} \frac{1}{\nu-2} \sum_{i_{1} \ldots i_{n}} g_{i_{1} \ldots i_{n-1}}^{0} n_{i_{1}}^{0} \ldots n_{i_{n}}^{0}\left(e_{i_{1}}+\ldots+e_{i_{n-1}}\right)^{2} e_{i_{n}}^{2}
\end{aligned}
$$

where we have only shown the pole part of $G_{\nu}(\mathbf{0})$. Since the bare coupling $g_{i_{1} \ldots i_{n-1}}^{0}$ contains in general a pole in $\nu-2$ of order $n-2$, this term produces a pole contribution of order $n-1$ to be cancelled by $I_{g_{i_{1} \ldots i_{n}}}^{(n-1)}$. Moreover, there are other pole contributions to $\ln \mathcal{Z}$ generated by $(n-1)$-loop diagrams without induced couplings and, consequently, the cancellation of pole terms requires that $g_{i_{1} \ldots i_{n}}^{0}$ have the general form

$$
\begin{aligned}
g_{i_{1} \ldots i_{n}}^{0}= & \pi \mu^{\nu-2} \frac{1}{\nu-2} \frac{1}{n}\left(g_{i_{1} \ldots i_{n-1}}^{0}\left(e_{i_{1}}+\ldots+e_{i_{n-1}}\right)^{2} e_{i_{n}}^{2}+\text { permutations }\right) \\
& +\mu^{(n-1)(\nu-2)}\left[\sum_{k=1}^{n-1} \frac{R_{i_{1} \ldots i_{n}}^{(k)}}{(\nu-2)^{k}}+g_{i_{1} \ldots i_{n}}(\mu)\right]
\end{aligned}
$$

where $R_{i_{1} \ldots i_{n}}^{(k)}$ comes enterely from contributions not included into $I_{g_{i_{1} \ldots i_{n-1}}}^{(n-1)}$. Thus, the finite term at $\nu=2$ of the Laurent series for the renormalization group condition $d g_{i_{1} \ldots i_{n}}^{0} / d \mu=0$ gives rise to

$\mu \frac{d}{d \mu} g_{i_{1} \ldots i_{n}}(\mu)=-\frac{\pi}{n}\left[g_{i_{1} \ldots i_{n-1}}\left(e_{i_{1}}+\ldots+e_{i_{n-1}}\right)^{2} e_{i_{n}}^{2}+(n-1)\right.$ similar $]+\ldots$

By defining the input value $g_{a} \equiv-1 / 2$, this equation recursively determines the leading logarithmic contribution to the induced couplings when $\mu$ is of order $\kappa_{0}$ and the integration constants are of order $\lambda^{-1}$. Up to three-loop order, the leading-log 
pieces of the partition function are

$$
\begin{aligned}
\frac{\ln \mathcal{Z}(\mu)}{\mathcal{A}}= & \sum_{a} n_{a}^{0}-\frac{\pi}{2} \ln \left(\kappa_{0} \lambda\right) \sum_{a, b} \beta^{2} n_{a}^{0} n_{b}^{0} e_{a}^{2} e_{b}^{2} \\
& +\frac{\pi^{2}}{4} \ln ^{2}\left(\kappa_{0} \lambda\right) \sum_{a, b, c} \beta^{4} n_{a}^{0} n_{b}^{0} n_{c}^{0} e_{a}^{2} e_{b}^{2} e_{c}^{2}\left(e_{a}+e_{b}\right)^{2} \\
& -\frac{\pi^{3}}{12} \ln ^{3}\left(\kappa_{0} \lambda\right) \sum_{a, b, c, d} \beta^{6} n_{a}^{0} n_{b}^{0} n_{c}^{0} n_{d}^{0} e_{a}^{2} e_{b}^{2} e_{c}^{2} e_{d}^{2}\left(e_{a}+e_{b}\right)^{2}\left(e_{a}+e_{b}+e_{c}\right)^{2}+\ldots
\end{aligned}
$$

In the case of one-component plasma the series of leading logs precisely agrees with the asymptotic expansion for the incomplete $\Gamma\left(0, z^{-1}\right)$ function, with $z=$ $\pi n^{0} e^{4} \beta^{2} \ln \left(\kappa_{0} \lambda\right)$. The result is

$$
\begin{aligned}
\frac{\ln \mathcal{Z}(\mu)}{\mathcal{A}} & =n^{0}+\frac{n^{0}}{2} \sum_{j=1}^{\infty}(-1)^{j} j !\left(\pi n^{0} e^{4} \beta^{2} \ln \left(\kappa_{0} \lambda\right)\right)^{j} \\
& =\frac{n^{0}}{2}\left[1+\frac{1}{z} \exp \left(\frac{1}{z}\right) \Gamma\left(0, \frac{1}{z}\right)\right],
\end{aligned}
$$

that produces the leading-log pressure expressed in term of the physical number density $\frac{\beta p}{\bar{n}}-1=\frac{1}{2} \bar{z}-\bar{z}^{2}+\frac{5}{2} \bar{z}^{3}-11 \bar{z}^{4}+\frac{137}{2} \bar{z}^{5}-510 \bar{z}^{6}+4341 \bar{z}^{7}+O\left(\bar{z}^{8}\right)$,

where $\bar{z}=\pi \bar{n} e^{4} \beta^{2} \ln \left(\bar{\kappa}_{0} \lambda\right)$. Finally, within this approximation the internal energy density becomes

$$
u=2 p-\frac{\bar{n}}{\beta} .
$$

\section{Conclusion}

With Brown and Yaffe as a guide, we have shown how the technique of effective field theory can be used in order to compute the partition function of the classical twodimensional plasma interacting via a $1 / r$ potential. Renormalization in the effective theory is essential already to one-loop level, and two-body induced couplings are required to order $e^{4}$. We have performed in detail the computation of the finite part of these couplings by exploiting the properties of the Coulomb Green's function in momentum space. We have derived explicit expressions for the one-loop equilibrium properties, without restriction on the quantum parameter $\eta_{a b}=\beta e_{a} e_{b} / \lambda_{a b}$. The higher order induced couplings satisfy renormalization group equations which can be solved in the leading logarithmic approximation. In the case of one-component plasma, we have been able to derive the asymptotic series of leading logs for the partition function in terms of the incomplete Gamma function.

\section{Acknowledgments}

This work was supported in part by the Spanish Ministry of Science and Technology (Grant FPA 2002-02037) and the University of the Basque Country (Grant 9/UPV00172.310-14497/2002). 


\section{Appendix. Computation of $F_{ \pm}(\boldsymbol{k})$}

In the following computation of $F_{ \pm}(\boldsymbol{k})$, we shall temporarily omit the indices $a, b$. We start with the evaluation of $F_{+}(\boldsymbol{k})$ given by the contour integral of the Green's function $G=(H-E)^{-1}$

$$
F_{+}(\boldsymbol{k})=\int_{C} \frac{d E}{2 \pi i} e^{-\beta E} \int d^{2} \boldsymbol{r} e^{-i \boldsymbol{k} \cdot \boldsymbol{r}}\left\langle\boldsymbol{r}\left|\frac{1}{H-E}\right| \boldsymbol{r}\right\rangle,
$$

where the contour $C$ encircles clockwise the cut along the positive real $E$ axis and all the bound-state poles when $e^{2}<0$ in the case of an attractive potential. To exploit the rotational symmetry of the problem and the properties on momentum space, it proves convenient to average over the angle of $\boldsymbol{k}$ and compute the spatial integral making use of

$$
\int_{0}^{\infty} d r r J_{0}(k r) J_{0}\left(\left|\boldsymbol{p}-\boldsymbol{p}^{\prime}\right| r\right)=\frac{1}{k} \delta\left(k-\left|\boldsymbol{p}-\boldsymbol{p}^{\prime}\right|\right) .
$$

This produces the expression

$$
F_{+}(\boldsymbol{k})=\int_{C} \frac{d E}{2 \pi i} e^{-\beta E} G_{+}(k, E),
$$

where

$$
G_{+}(k, E)=\int d^{2} \boldsymbol{p} d^{2} \boldsymbol{p}^{\prime}\left\langle\boldsymbol{p}\left|\frac{1}{H-E}\right| \boldsymbol{p}^{\prime}\right\rangle \frac{1}{2 \pi k} \delta\left(k-\left|\boldsymbol{p}-\boldsymbol{p}^{\prime}\right|\right) .
$$

The key to the computation of $G_{+}(k, E)$ is the closed form formula for the momentum space Green's function $G\left(\boldsymbol{p}, \boldsymbol{p}^{\prime}\right) \equiv\left\langle\boldsymbol{p}\left|(H-E)^{-1}\right| \boldsymbol{p}^{\prime}\right\rangle$ of the two-dimensional Coulomb problem. This form has been given in [8] following the clever treatment of Schwinger [7]. Let us summarize the main results concerning this Green's function. The momentum representation equation for the Green's function is

$$
\left(\frac{\boldsymbol{p}^{2}}{2 m}-E\right) G\left(\boldsymbol{p}, \boldsymbol{p}^{\prime}\right)+\frac{e^{2}}{2 \pi} \int d^{2} \boldsymbol{p}^{\prime \prime} \frac{1}{\left|\boldsymbol{p}-\boldsymbol{p}^{\prime \prime}\right|} G\left(\boldsymbol{p}^{\prime \prime}, \boldsymbol{p}^{\prime}\right)=\delta\left(\boldsymbol{p}-\boldsymbol{p}^{\prime}\right) \text {. }
$$

Based on the (conformal) correspondence between the points of the euclidean twodimensional momentum space and the surface of the unit three-dimensional sphere

$$
\begin{aligned}
& p_{x}=\frac{p_{0}}{2(\cos \theta / 2)^{2}} \sin \theta \cos \phi, \\
& p_{y}=\frac{p_{0}}{2(\cos \theta / 2)^{2}} \sin \theta \sin \phi,
\end{aligned}
$$

the element of area on the sphere and the two-dimensional measure in the plane are related by

$$
d \Omega=\left(\frac{2 p_{0}}{p_{0}^{2}+p^{2}}\right)^{2} d^{2} \boldsymbol{p}
$$

where $p_{0}=\sqrt{-2 m E}$ is the positive real wavenumber corresponding to the negative energy $E$. The angular distance $\gamma$ between the orientations of the two points $\Omega=(\theta, \phi)$, 
$\Omega^{\prime}=\left(\theta^{\prime}, \phi^{\prime}\right)$ on the unit sphere is related to the euclidean distance between the corresponding points $\boldsymbol{p}$ and $\boldsymbol{p}^{\prime}$ by

$$
\left(2 \sin \frac{\gamma}{2}\right)^{2}=\frac{4 p_{0}^{2}}{\left(p_{0}^{2}+p^{2}\right)\left(p_{0}^{2}+p^{\prime 2}\right)}\left|\boldsymbol{p}-\boldsymbol{p}^{\prime}\right|^{2} .
$$

Then, noting that the term $\left|\boldsymbol{p}-\boldsymbol{p}^{\prime \prime}\right|^{-1}$ can be written as

$$
\frac{1}{\left|\boldsymbol{p}-\boldsymbol{p}^{\prime \prime}\right|}=2 p_{0}\left(p_{0}^{2}+p^{2}\right)^{-1 / 2}\left(p_{0}^{2}+p^{\prime \prime 2}\right)^{-1 / 2} \sum_{l, m} \frac{4 \pi}{2 l+1} Y_{l}^{m}(\Omega) Y_{l}^{m}\left(\Omega^{\prime \prime}\right)^{*},
$$

and the completeness relation of the spherical harmonics

$$
\delta\left(\Omega-\Omega^{\prime}\right)=\sum_{l, m} Y_{l}^{m}(\Omega) Y_{l}^{m}\left(\Omega^{\prime}\right)^{*},
$$

the Green's function is just

$$
G\left(\boldsymbol{p}, \boldsymbol{p}^{\prime}\right)=8 m p_{0}^{2}\left(p_{0}^{2}+p^{2}\right)^{-3 / 2}\left(p_{0}^{2}+p^{\prime 2}\right)^{-3 / 2} \sum_{l, m} \frac{Y_{l}^{m}(\Omega) Y_{l}^{m}\left(\Omega^{\prime}\right)^{*}}{1+\frac{\nu}{l+1 / 2}},
$$

where

$$
\nu=\frac{e^{2} m}{p_{0}}
$$

is positive when the potential is repulsive. For an attractive potential $(\nu<0)$, the poles corresponding to the bound states are

$$
E_{l}=-\frac{m e^{4}}{2(l+1 / 2)^{2}} .
$$

The next step is to express the remaining factor in the integrand of (A.4) in terms of the spherical harmonics associated with $\boldsymbol{p}$ and $\boldsymbol{p}^{\prime}$. This is readily performed from the series of Legendre polynomials

$$
\delta\left(2-2 \cos \gamma-a^{2}\right)=\sum_{l=0}^{\infty} \frac{2 l+1}{4} P_{l}\left(1-\frac{a^{2}}{2}\right) P_{l}(\cos \gamma),
$$

with the identification

$$
a^{2}=\frac{4 p_{0}^{2} k^{2}}{\left(p_{0}^{2}+p^{2}\right)\left(p_{0}^{2}+p^{\prime 2}\right)},
$$

where $\gamma$ is the angle between the orientations in the unit sphere corresponding to $\boldsymbol{p}$ and $\boldsymbol{p}^{\prime}$. Therefore, using the addition of spherical harmonics

$$
P_{l}(\cos \gamma)=\frac{4 \pi}{2 l+1} \sum_{m=-l}^{l} Y_{l}^{m}(\Omega) Y_{l}^{m}\left(\Omega^{\prime}\right)^{*},
$$

one obtains the required formula

$$
\begin{aligned}
\frac{1}{2 \pi k} \delta\left(k-\left|\boldsymbol{p}-\boldsymbol{p}^{\prime}\right|\right) & =\frac{4 p_{0}^{2}}{\left(p_{0}^{2}+p^{2}\right)\left(p_{0}^{2}+p^{\prime 2}\right)} \sum_{l, m} P_{l}\left(1-\frac{a^{2}}{2}\right) Y_{l}^{m}(\Omega) Y_{l}^{m}\left(\Omega^{\prime}\right)^{*} \\
& =\left(\frac{2 p_{0}}{p_{0}^{2}+p^{2}}\right)^{2} \delta\left(\Omega-\Omega^{\prime}\right)+\mathrm{O}\left(k^{2}\right) .
\end{aligned}
$$


With these results in hand, we return to the computation of $G_{+}(k, E)$. Using the integral representation of Legendre polynomials [10]

$$
P_{l}(\cos \gamma)=\frac{1}{\pi} \int_{0}^{\pi} d \phi(\cos \gamma+i \sin \gamma \cos \phi)^{l}
$$

and the hypergeometric series with $b=\nu+1 / 2$ and $z=\cos \gamma+i \sin \gamma \cos \phi$

$$
\sum_{l=0}^{\infty} \frac{z^{l}}{l+b}=F(b, 1, b+1, z), \quad(|z|<1)
$$

the sum over $l$ in the Green's function (A.12) can be easily written in an appropriate form to study the $\boldsymbol{k} \rightarrow 0$ or, equivalently, the $\gamma \rightarrow 0$ behavior. This form is based on the following transformation formula for $F(b, 1, b+1, z)$, valid when $|\arg (1-z)|<\pi$ and $|1-z|<1$ (see, for example, p 559 of [10])

$F(b, 1, b+1, z)=b \sum_{n=0}^{\infty} \frac{(b)_{n}}{n !}[\psi(n+1)-\psi(b+1)-\ln (1-z)](1-z)^{n}$.

Therefore, by evaluating the $\phi$ integral of the $n=0$ term, we obtain

$$
\sum_{l=0}^{\infty} \frac{P_{l}(\cos \gamma)}{l+\nu+1 / 2}=-\gamma_{\mathrm{E}}-\psi\left(\frac{1}{2}+\nu\right)-\ln \left(\frac{\gamma}{2}\right)+O(\gamma \ln \gamma)
$$

where $\gamma_{\mathrm{E}}$ is the Euler's constant and $\psi$ denotes the digamma function. Also, we need

$$
\sum_{l=0}^{\infty} P_{l}(\cos \gamma)=\frac{1}{2 \sin \gamma / 2}
$$

Putting all pieces together, the non-vanishing portion of $G_{+}(k, E)$ as $\boldsymbol{k} \rightarrow 0$ is readily found by the integration of (A.4). This yields, after subtraction of the $e^{2}=0$ contribution, the result

$$
\begin{aligned}
G_{+}(k, E)= & -\frac{2 m \nu}{p_{0} k}-\frac{m \nu^{2}}{p_{0}^{2}}\left[2 \gamma_{\mathrm{E}}-1+2 \ln \left(\frac{k}{p_{0}}\right)+2 \psi\left(\frac{1}{2}+\nu\right)\right]+O(k) \\
= & \beta\left\{-\frac{2 \pi y}{\lambda k(-t)}-\frac{\pi y^{2}}{2(-t)^{2}}\left[2 \gamma_{\mathrm{E}}-1+\ln \left(\frac{\lambda^{2} k^{2}}{4 \pi}\right)\right.\right. \\
& \left.\left.-\ln (-t)+2 \psi\left(\frac{1}{2}+\frac{\sqrt{\pi} y}{\sqrt{-t}}\right)\right]+O(k)\right\},
\end{aligned}
$$

where $t=\beta E$, and the dimensionless parameter $y$ is the ratio of the Coulomb energy for two particles separated by one thermal (reduced) wavelength to the temperature

$$
y= \pm \sqrt{\frac{\beta m e^{4}}{2 \pi}} \Leftrightarrow \eta_{a b}=\frac{\beta e_{a} e_{b}}{\lambda_{a b}}
$$

Thus, the \pm sign accounts for the repulsive $(+)$ or attractive $(-)$ cases. Note that $\arg (-t)$ is purely real when $t$ is on the negative part of the real $t$ axis, so that, the convention is $-\pi<\arg (-t)<\pi$ when $0<\arg (t)<2 \pi$.

It remains to perform the required contour integral. The result $F_{+}(k)$ parametrically depends on $y$, and we will distinguish between the small and large $|y|$ 
limits. For both the attractive and repulsive cases, the small $|y|<<1$ limit can be obtained from the power series expansion of the $\psi$ function

$\psi(1 / 2+z)=-\gamma_{\mathrm{E}}-2 \ln 2+\sum_{n=2}^{\infty} \zeta(n)(-1)^{n}\left(2^{n}-1\right) z^{n-1}, \quad\left(|z|<\frac{1}{2}\right)$

provided that the contour $C$ cuts the real $t$ axis at the left of $t=-4 \pi y^{2}$, in the case of an attractive potential, or at the left of $t=0$, in the case of a repulsive potential. The insertion term by term of this series in the Hankel's expression for $\Gamma(z)$

$$
\frac{1}{\Gamma(z)}=\int_{C} \frac{d t}{2 \pi i}(-t)^{-z} e^{-t}
$$

and its derivative with respect to $z$

$$
\frac{\psi(z)}{\Gamma(z)}=\int_{C} \frac{d t}{2 \pi i} \ln (-t)(-t)^{-z} e^{-t}
$$

produces the final result, after subtraction of the $e^{2}=0$ contribution

$$
\Delta F_{+}(\boldsymbol{k})=-\frac{2 \pi y}{\lambda k}-\frac{\pi y^{2}}{2}\left[\gamma_{\mathrm{E}}+\ln \left(\frac{\lambda^{2} k^{2}}{64 \pi}\right)+f(y)\right]+O(k),
$$

where the function $f(y)$ is defined by the power series expansion

$$
f(y)=-2-2 \sum_{n=2}^{\infty} \frac{\zeta(n)\left(2^{n}-1\right)}{\Gamma((3+n) / 2)}(-\sqrt{\pi} y)^{n-1}=-2+\frac{4 \pi^{2} y}{3}-7 \pi \zeta(3) y^{2}+O(y)^{3} .
$$

Note that here $y$ can be positive or negative.

Now, consider the asymptotic behavior when $|y| \rightarrow \infty$. For the attractive case, the leading contribution comes from the lowest bound state, as it is easily noted from the dependence on $e^{-\beta H}$. Hence, all we need is the residue $-e^{4 \pi y^{2}}$ of the integrand $e^{-t} G_{+}(k, E)$ at the bound state pole $t=-4 \pi y^{2}$. Thus, when $y \rightarrow-\infty$,

$$
\Delta F_{+}(\boldsymbol{k}) \sim e^{4 \pi y^{2}}
$$

and

$$
f(y) \sim-\frac{2}{\pi y^{2}} e^{4 \pi y^{2}} .
$$

To obtain the leading asymptotic behavior in the case of strong repulsion, it proves convenient to study separately the contribution counterclockwise around the ramification point $t=0$, and the contribution from the discontinuity of $G_{+}(k, E)$ at the cut along the positive real axis. As we shall see, the discontinuity yields exponentially small corrections. Using the identity

$$
\operatorname{Im} \psi(1 / 2+i u)=\frac{\pi}{2} \tanh (\pi u)
$$

and noting that Disc $\ln (-t)=-2 \pi i$, the discontinuity of $G_{+}(k, E)$ becomes

$$
\begin{aligned}
G_{+}(k, E+i \varepsilon)-G_{+}(k, E-i \varepsilon) & =\frac{i \pi^{2} y^{2}}{t^{2}}\left[-1-\tanh \left(\frac{\pi^{3 / 2} y}{\sqrt{t}}\right)\right] \\
& =-\frac{2 i \pi^{2} y^{2}}{t^{2}}+\frac{i \pi^{2} y^{2}}{t^{2}}\left[1-\tanh \left(\frac{\pi^{3 / 2} y}{\sqrt{t}}\right)\right],(t>0)(\text { A. } 34)
\end{aligned}
$$


The last term in the second line produces an integral well defined for $y>0$, which can be studied by inserting the representation of $\tanh z$ as a series of exponentials. The resulting expression contributing to $\Delta F_{+}(\boldsymbol{k})$ has the form

$$
\begin{gathered}
-\pi y^{2} \sum_{n=0}^{\infty}(-1)^{n} \int_{0}^{\infty} \frac{d t}{t^{2}} \exp \left(-t-\frac{2(n+1) \pi^{3 / 2} y}{\sqrt{t}}\right) \\
\sim-\frac{2 y}{\sqrt{3}} e^{-3 \pi y^{2 / 3}}\left(1+\frac{17}{36 \pi y^{2 / 3}}+\ldots\right)
\end{gathered}
$$

where, as $y \rightarrow \infty$, the leading behavior corresponds to the first term in the sum, which has been evaluated by the steepest descent approximation. The first term in (A.34) yields

$$
-\pi y^{2} \int_{\delta}^{\infty} \frac{d t}{2 \pi} \frac{e^{-t}}{t^{2}}=-\frac{\pi y^{2}}{\delta}+\pi y^{2}\left(1-\gamma_{\mathrm{E}}-\ln \delta\right)+O(\delta) .
$$

It remains to compute the contribution from the small circle around the origin. There, $-t=\delta e^{i \theta}$, with $-\pi<\theta<\pi$, and $\ln (-t)=\ln \delta+i \theta$. Then, the contribution to $\Delta F_{+}(\boldsymbol{k})$ may be obtained by using the large $z$ behavior of the $\psi$ function

$$
\psi(1 / 2+z) \sim \ln z+\sum_{n=1}^{\infty} \frac{B_{2 n}}{2 n}\left(1-2^{1-2 n}\right) z^{-2 n}, \quad(|\arg (z)|<\pi)
$$

where $B_{2 n}$ are the Bernoulli numbers. The angular integral of $e^{-t} G_{+}(k, E)$ produces singular terms which serve to cancel the linear and logarithmic divergences in (A.36), in addition to non-vanishing terms when $\delta \rightarrow 0$. Putting the pieces together, one immediately finds all terms of $\Delta F_{+}(\boldsymbol{k})$ non-exponentially suppressed in the large $y$ limit,

$$
\Delta F_{+}(\boldsymbol{k}) \sim-\frac{\pi y^{2}}{2}\left[4 \gamma_{\mathrm{E}}-3+\ln \left(\frac{\lambda^{2} k^{2} y^{2}}{4}\right)\right]-\frac{2 \pi y}{\lambda k}-\frac{1}{24} .
$$

Note that the only contribution of the asymptotic expansion of the $\psi(1 / 2+z)$ function comes from the logarithmic and the $z^{-2}$ terms. With this result, the asymptotic behavior of $f(y)$ as $y \rightarrow \infty$ is given by

$$
f(y) \sim \ln \left(16 \pi y^{2}\right)+3 \gamma_{\mathrm{E}}-3+\frac{1}{12 \pi y^{2}} .
$$

It remains to compute the exchange contribution. The nonvanishing portion at $\boldsymbol{k}=0$ of the exchange contribution is given by

$$
F_{-}(\mathbf{0})=\int_{C} \frac{d E}{2 \pi i} e^{-\beta E} G_{-}(0, E)
$$

where

$$
G_{-}(0, E)=\int d^{2} \boldsymbol{p} G(\boldsymbol{p},-\boldsymbol{p}) .
$$

Noting that the orientations on the unit sphere of the points $\boldsymbol{p}$ and $-\boldsymbol{p}$ are $\Omega=(\theta, \phi)$ and $\Omega^{\prime}=(\theta, \phi+\pi)$ respectively, the angular distance between them is $2 \theta$. Thus, using (A.17), the sum over $m$ in (A.12) gives a factor $P_{l}(\cos 2 \theta)$. Now, the integration over $\boldsymbol{p}$ can be performed with the aid of the following integrals of Legendre polynomials

$$
\int_{0}^{\pi} d \theta \sin \theta P_{l}(\cos 2 \theta) \cos ^{2} \theta / 2=\frac{(-1)^{l}}{2 l+1}
$$


and, finally, the resulting series is summed using the series representation

$$
\sum_{l=0}^{\infty} \frac{(-1)^{l}}{2 l+1+2 \nu}=\frac{1}{4}\left[\psi\left(\frac{3}{4}+\frac{\nu}{2}\right)-\psi\left(\frac{1}{4}+\frac{\nu}{2}\right)\right] .
$$

Hence, subtracting the $e^{2}=0$ contribution, $G_{-}(\mathbf{0}, E)$ reads

$$
G_{-}(0, E)=\frac{m \nu}{2 p_{0}^{2}}\left[\psi\left(\frac{1}{4}+\frac{\nu}{2}\right)-\psi\left(\frac{3}{4}+\frac{\nu}{2}\right)\right] .
$$

Since the exchange contribution involves particles of the same species, only the computation of $F_{-}(\mathbf{0})$ in the repulsive case $y>0$ is required. In this case, we can use the integral representation

$$
\psi\left(\frac{3}{4}+z\right)-\psi\left(\frac{1}{4}+z\right)=\frac{1}{2} \int_{0}^{\infty} d u \frac{e^{-z u}}{\cosh u / 4} . \quad(\operatorname{Re} z>0)
$$

When $y \ll 1$, we insert the power series expansion of the exponential $e^{-z u}$ and perform the $u$ integral with the aid of

$$
\int_{0}^{\infty} d u \frac{u^{n}}{\cosh u / 4}=2 \Gamma(n+1)[\zeta(n+1,1 / 4)-\zeta(n+1,3 / 4)], \quad(\operatorname{Re} n>-1)(A
$$

where $\zeta(s, a)$ denotes the generalized Riemann zeta function. Then, the evaluation of the contour integral yields the power series expansion

$$
F_{-}(\mathbf{0})=-\frac{\pi y}{2}-\sum_{n=1}^{\infty} \frac{(-1)^{n}[\zeta(1+n, 1 / 4)-\zeta(1+n, 3 / 4)]}{2 \Gamma((3+n) / 2)}\left(\frac{\sqrt{\pi} y}{2}\right)^{n+1} .
$$

Similarly to the function $f$ for the direct part, it is useful to define the function $\widetilde{f}(y)$ as

$$
\widetilde{f}(y) \equiv-\frac{2}{\pi y^{2}} F_{-}(\mathbf{0})=\frac{1}{y}-4 C+\frac{\pi^{3} y}{3}+O\left(y^{2}\right),
$$

where $C$ is the Catalan's number.

Finally, the positive large $y$ limit may be obtained by evaluating the $u$ integral (A.45) using the Laplace's method of asymptotic analysis. Inserting the Taylor expansion of $(\cosh u / 4)^{-1}$ about $u=0$, produces a convergent series in $u<2 \pi$ which can be integrated term-by-term. Therefore, the Hankel's formula leads to

$$
F_{-}(\mathbf{0}) \sim-\frac{1}{4}
$$

and

$$
\widetilde{f}(y) \sim \frac{1}{2 \pi y^{2}},
$$

apart from exponentially small corrections.

\section{References}

[1] Brown L S and Yaffe L G 2001 Phys. Rep. 3401

[2] Ando T, Fowler A B and Stern F 1982 Rev. Mod. Phys. 54437

[3] Fetter A L 1974 Phys. Rev. B 103739

[4] Chalupa J 1975 Phys. Rev. B 124

Chalupa J 1976 Phys. Rev. B 132243 (erratum) 
[5] Totsuji H 1978 Phys. Rev. A 17399

[6] Gann R C, Chakravarty S and Chester G V 1979 Phys. Rev. B 20326

[7] Schwinger J 1964 J. Math. Phys. 51606

[8] Dittrich W 1999 Am. J. Phys. 67768

[9] Braaten E and Nieto A 1995 Phys. Rev. D 516990

[10] Abramowitz M and Stegun I A 1972 Handbook of Mathematical Functions (New York: Dover) p 559 